\title{
Experimental Distribution of Entanglement with Separable Carriers
}

\author{
A. Fedrizzi, ${ }^{1, *}$ M. Zuppardo, ${ }^{2}$ G. G. Gillett, ${ }^{1}$ M. A. Broome,${ }^{1}$ M. P. Almeida, ${ }^{1}$ M. Paternostro, ${ }^{3}$ \\ A. G. White, ${ }^{1}$ and T. Paterek ${ }^{2,4, \dagger}$ \\ ${ }^{1}$ Centre for Engineered Quantum Systems and Centre for Quantum Computer and Communication Technology, \\ School of Mathematics and Physics, University of Queensland, Brisbane, Queensland 4072, Australia \\ ${ }^{2}$ School of Physical and Mathematical Sciences, Nanyang Technological University, 637371 Singapore, Singapore \\ ${ }^{3}$ Centre for Theoretical Atomic, Molecular, and Optical Physics, School of Mathematics and Physics, \\ Queen's University Belfast, BT7 1NN, United Kingdom \\ ${ }^{4}$ Centre for Quantum Technologies, National University of Singapore, 117543 Singapore, Singapore
} (Received 15 June 2013; published 4 December 2013)

The key requirement for quantum networking is the distribution of entanglement between nodes. Surprisingly, entanglement can be generated across a network without direct transfer-or communication - of entanglement. In contrast to information gain, which cannot exceed the communicated information, the entanglement gain is bounded by the communicated quantum discord, a more general measure of quantum correlation that includes but is not limited to entanglement. Here, we experimentally entangle two communicating parties sharing three initially separable photonic qubits by exchange of a carrier photon that is unentangled with either party at all times. We show that distributing entanglement with separable carriers is resilient to noise and in some cases becomes the only way of distributing entanglement through noisy environments.

DOI: 10.1103/PhysRevLett.111.230504

PACS numbers: 03.67.Bg, 03.67.Hk, 42.50.Ex

Communication is the exchange of physical systems aimed at establishing correlations between communicating parties. The total correlations are quantified by the mutual information established between the sender and receiver [1], and information theory states that the gain in mutual information cannot exceed the amount of communicated information [2,3]:

$$
I_{\text {final }}-I_{\text {initial }} \leq I_{\text {comm }} .
$$

This statement holds true both in classical and quantum physics, but it does not generalize to quantum entanglement [4]. Entanglement is a purely nonclassical type of correlation enabling tasks such as quantum teleportation [5], secure cryptography [6], improved communication complexity [7], and quantum dense coding [8].

Remarkably, Cubitt et al. showed [9] that quantum entanglement can be distributed between remote parties without communicating it: through the exchange of a carrier system that is never entangled with sender or receiver. The gain in entanglement $\mathcal{E}$ between communicating sites is instead bounded from above $[3,10]$ by the amount of communicated quantum discord $\mathcal{D}_{\text {comm }}$

$$
\mathcal{E}_{\text {final }}-\mathcal{E}_{\text {initial }} \leq \mathcal{D}_{\text {comm }}
$$

Quantum discord is a type of nonclassical correlation $[11,12]$ which equates to entanglement in pure quantum states but can persist in mixed states with zero entanglement. Equation (2) implies that discord is a necessary resource for entanglement distribution with separable carriers. This provides an alternative method to conventional protocols which aim at entangling quantum nodes via transfer of preavailable entanglement $[13,14]$.

Here, we experimentally demonstrate entanglement distribution via separable carriers using polarization-encoded single photons. We validate the discord bound in Eq. (2) and show, both in theory and practice, that the implemented entanglement distribution protocol [15] is robust against noise, despite distributing only a small amount of entanglement with each carrier. This is a crucial requirement for practical entanglement distribution between the nodes of a quantum network in a noisy environment. Moreover, we show the existence of a significant range of parameters for which the use of our protocol based on the communication of separable carriers is the only way of establishing entanglement remotely.

A typical communication scenario is depicted in Fig. 1. Two parties, Alice and Bob, hold quantum systems $A$ and $B$, respectively. Alice uses a carrier quantum system $C$, which encodes her communication to Bob. We evaluate the entanglement gain between their respective laboratories [Eq. (2)] using $\mathcal{E}_{\text {final }}=\mathcal{E}_{A: C B}, \quad \mathcal{E}_{\text {initial }}=\mathcal{E}_{A C: B}$, and $\mathcal{D}_{\text {comm }}=\mathcal{D}_{A B \mid C}$. Here, $\mathcal{E}_{X: Y}$ denotes the relative entropy of entanglement between $X$ and $Y$ [16], while $\mathcal{D}_{X \mid Y}$ is the relative entropy of discord [17], also known as the one-way quantum deficit [18].

In this protocol, the two-level systems $A$ and $B$ are prepared in a separable state $\alpha_{A B}$ that is a mixture of the four Bell states $\left|\psi_{ \pm}\right\rangle_{A B}=(1 / \sqrt{2})(|01\rangle \pm|10\rangle)_{A B}$ and $\left|\phi_{ \pm}\right\rangle_{A B}=$ $(1 / \sqrt{2})(|00\rangle \pm|11\rangle)_{A B}$, with probability of occurrence $p_{\psi_{ \pm}}$ and $p_{\phi_{+}}$, respectively. Such a state is separable if and only if the highest probability in the mixture does not exceed $50 \%$ 


\section{0}

$\circ$

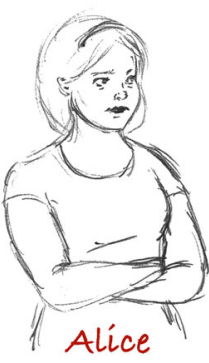

$\odot$

(1)
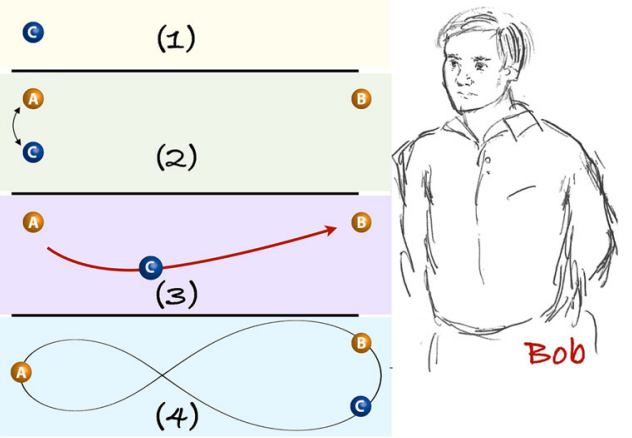

FIG. 1 (color online). (Quantum) communication scenario. Alice locally interacts her system $A$ with the carrier system $C$, which is then sent to Bob's site. It is possible to establish entanglement between their respective laboratories even though there was no initial entanglement between them and no entanglement is communicated. This is accomplished as follows: In step (1), the fully separable initial state of the three systems is prepared. In step (2), Alice applies a suitable operation on $A$ and $C$, which keeps the latter separable from the rest of the systems but creates entanglement between $A$ and joint systems made out of $B$ and $C$ together. In step (3), the unentangled carrier $C$ is transmitted to Bob. As shown in panel (4), this establishes entanglement between the laboratories of Alice and Bob.

[19]. The two-level carrier $C$ is initially with Alice and uncorrelated from the other systems, so that the overall initial state is taken as $\alpha=\alpha_{A B} \otimes \alpha_{C}$. Here, $\alpha_{C}=(1 / 2) \times$ $\left(\mathbb{1}+c_{x} \sigma_{x}\right)$ with $\mathbb{1}$ the identity matrix, $\sigma_{k}(k=x, y, z)$ the Pauli $k$ matrix, and $c_{x} \in[-1,1]$.

Alice now generates the state $\beta=\mathcal{P}_{A C} \alpha \mathcal{P}_{A C}^{\dagger}$ by applying a controlled-phase gate $\mathcal{P}_{A C}=|0\rangle\left\langle\left. 0\right|_{A} \otimes \mathbb{1}_{C}+\mid 1\right\rangle \times$ $\left\langle\left. 1\right|_{A} \otimes \sigma_{z, C}\right.$ on her systems. We want the carrier qubit to remain separable from the other systems $\mathcal{E}_{A B: C}(\beta)=0$, while system $A$ should become entangled with the subsystem composed of $B$ and $C$; i.e., we require $\mathcal{E}_{A: C B}(\beta)>0$. Finally, carrier $C$ is transmitted to Bob and, as a result, the laboratories of Alice and Bob share entanglement.

We choose the initial states of $A$ and $B$ such that $C$ stays separable while maximizing the entanglement in the $A \mid C B$ bipartition at the conclusion of the protocol. A possible instance is given by the $A B$-separable state

$$
\begin{aligned}
\alpha_{A B}= & \frac{1}{4} \sum_{j=0}^{1}\left|z_{j} z_{j}\right\rangle\left\langle z_{j} z_{j}\left|+\frac{1}{8} \sum_{j=0}^{1}\right| x_{j} x_{j}\right\rangle\left\langle x_{j} x_{j}\right| \\
& +\frac{1}{8} \sum_{j=0}^{1}\left|y_{j} y_{1-j}\right\rangle\left\langle y_{j} y_{1-j}\right|,
\end{aligned}
$$

which is a mixture of two-qubit states formed by the eigenstates $\left|k_{j}\right\rangle$ of Pauli operators $\sigma_{k}$, with eigenvalue $(-1)^{j}$. As a witness of entanglement, we use $\lambda_{X \mid Y}^{\min }$, the minimum eigenvalue of the partial transposition of the bipartite density matrix $\beta_{X \mid Y}$ with respect to subsystem $X$ [20]. Since the theoretical states considered and experimental states measured yield at most one negative eigenvalue, this witness is related to the negativity $\mathcal{N}$ [21] —an entanglement measure-by $\mathcal{N}_{X \mid Y}=\left(\left|\lambda_{X \mid Y}^{\min }\right|-\lambda_{X \mid Y}^{\min }\right) / 2$.

Within the class of initial states $\alpha$ on which one applies $\mathcal{P}_{A C}$, the state composed of Eq. (3) and $\alpha_{C}$ with $c_{x}=-\frac{1}{2}$ gives $\mathcal{N}_{A \mid B C}=1 / 16=0.0625$, the highest possible amount of entanglement that can be distributed via separable states [15]. We focus on $\mathcal{N}$, as its presence in $\beta$ guarantees that (i) the entanglement established between Alice and Bob can be localized into entanglement between $A$ and $B$ using only local operations performed at Bob's site [3] and (ii) such localized entanglement is distillable [22]. Therefore, by repeating this protocol a sufficient number of times and performing entanglement distillation, one can in principle obtain maximally entangled pairs between Alice and Bob without ever communicating entanglement between them.

The circuit diagram in Fig. 2(a) shows the conceptual implementation of the protocol, while the experimental setup is shown in Fig. 2(b). Using four single photonsone of which acts as a trigger with the other three as the qubits $A, B$, and $C$-we prepare the state $\alpha_{A B}$ by summing up the individual pure-state terms in Eq. (3), with measurement acquisition times corresponding to the weights. A similar technique prepares the mixed state $\alpha_{C}$ and

(a)

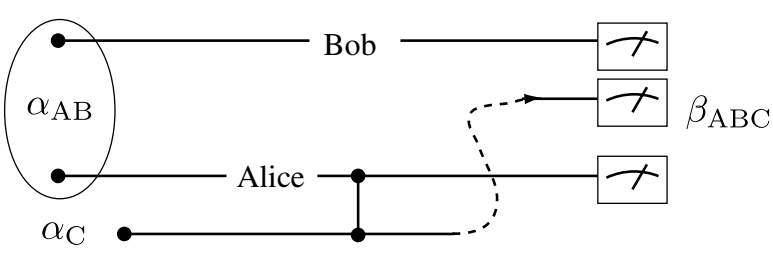

(b)

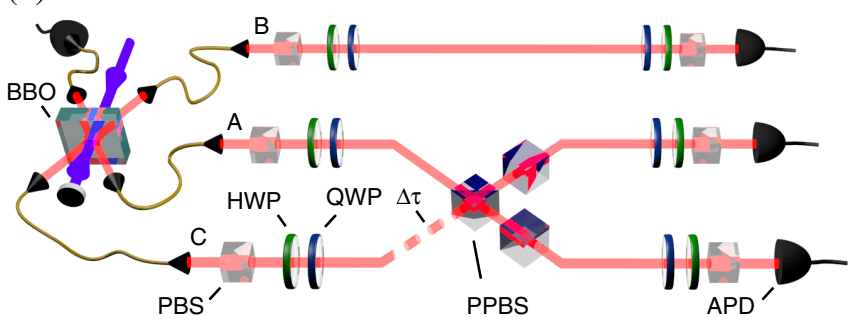

FIG. 2 (color online). Entanglement distribution scheme. (a) Equivalent quantum circuit diagram for our protocol. (b) Two pairs of single photons at $820 \mathrm{~nm}$ are created via spontaneous parametric down-conversion in a $\beta$-barium borate crystal (BBO) pumped by a frequency-doubled femtosecond Ti: sapphire laser. One photon serves as a trigger, while the other three are initialized with polarizing beam splitters (PBS) and half-wave (HWP) and quarter-wave plates (QWP). The photons representing systems $A$ and $C$ are subjected to a probabilistic controlled-phase gate based on two-photon interference at a partially polarizing beam splitter (PPBS) [23]. Projective measurements are carried out with a combination of HWPs, QWPs, and PBSs, before the photons are detected by singe-photon avalanche photodiodes (APD) connected to a coincidence logic. 
guarantees that the initial state $\alpha_{A B C}$ is separable. Systems $A$ and $C$ interact in a controlled-phase gate [23], before the output state $\beta_{A B C}$ is characterized through a three-qubit state tomography [24]. The total integration time was $387 \mathrm{~h}$, during which we counted $\sim 30000$ fourfold coincidence events. The reconstructed density matrix has a large overlap with the ideal state, quantified by a fidelity of $\mathcal{F}\left(\beta_{\text {exp }}, \beta_{\text {ideal }}\right) \equiv \operatorname{Tr}\left[\left(\beta_{\text {exp }}^{1 / 2} \beta_{\text {ideal }} \beta_{\text {exp }}^{1 / 2}\right)^{1 / 2}\right]^{2}=0.98$, and is shown in Fig. 3. To estimate the uncertainty, we perform a Monte Carlo analysis based on 10000 Poissoniandistributed variations of the measured photon counts. The corresponding population of reconstructed density matrices is used to evaluate an average fidelity of $\mathcal{F}_{\text {est }}=$ $0.967 \pm 0.007$, which is extremely close to the experimental value.

In order to experimentally study the resilience of the protocol against noise and to obtain an unambiguous signature for entanglement distribution with separable states, we add increasing amounts of white noise to the initial state, thus obtaining $\tilde{\alpha}_{A B C}=(1-p) \alpha_{A B C}+(p / 8) \mathbb{1}$. Previously, this method has been used to assess the generation of bound-entangled states [25]. Theoretically, $\tilde{\alpha}_{A B C}$ allows entanglement distribution with separable carriers for all $p<\frac{1}{3}$.

In Fig. 4(a) we plot $\lambda^{\text {min }}$ for all bipartitions of the measured states as a function of added white noise. For $p=0$, only bipartition $A \mid B C$ is entangled, indicating a successful demonstration of the protocol. However, as shown in Fig. 4(c), only 17.4\% of the Monte Carlo population has the required success signature. This proportion rises rapidly with the addition of small amounts of white
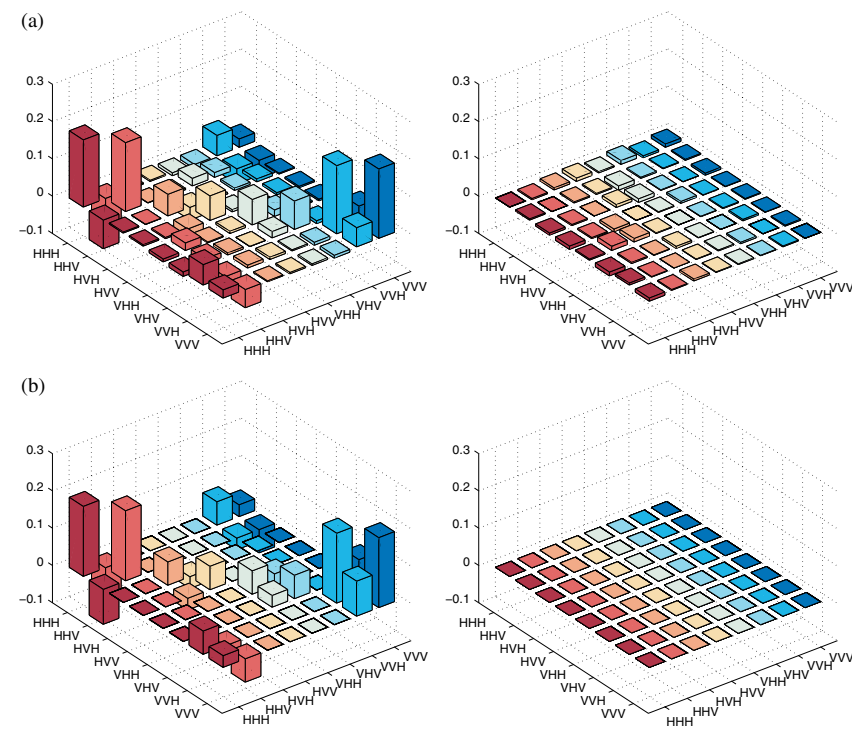

FIG. 3 (color online). Real (left) and imaginary (right) parts of the output state $\beta_{A B C}$. (a) Experimental density matrix, obtained via three-qubit state tomography. (b) Ideal density matrix. The fidelity between (a) and (b) is $98 \%$, see text. noise: $96.5 \%$ of the population successfully demonstrates the protocol for $p=0.1667$.

The measured negativity with the maximum added noise $(p=0.1667)$ is $\mathcal{N}_{A \mid B C}^{\exp }=0.0172$ with $\mathcal{N}_{B \mid A C}^{\exp }=$ $\mathcal{N}_{C \mid A B}^{\exp }=0$. In order to exclude the possibility that the controlled-phase gate introduces bound entanglement [26] that is distributed by system $C$, we provide an explicit decomposition of the experimental states in terms of convex sums of product states of the $C \mid A B$ bipartition in Secs. I and II of the Supplemental Material [27]. We further show in Sec. IV of [27] that the experimentally distributed (a)

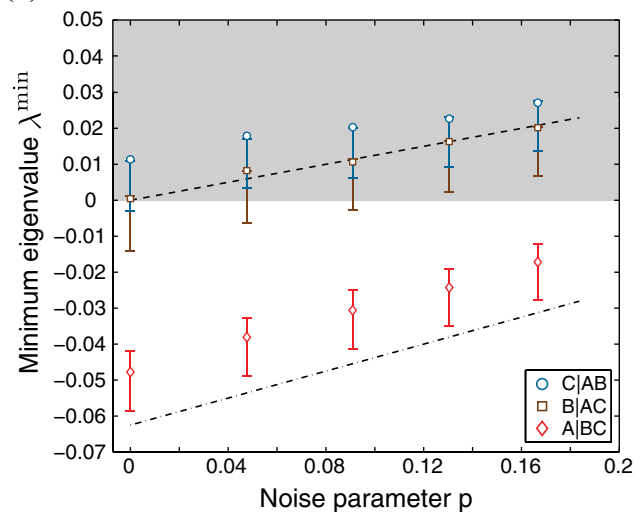

(c)

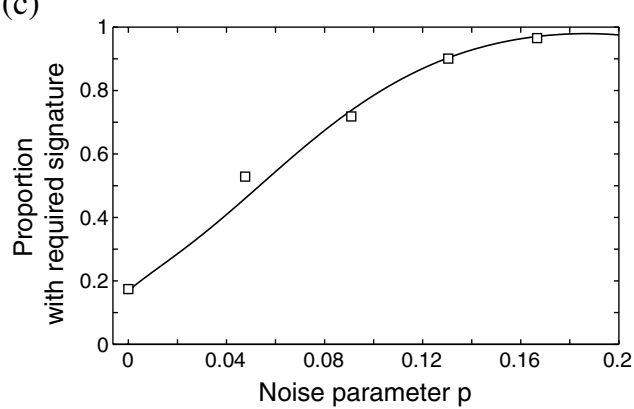

(b)

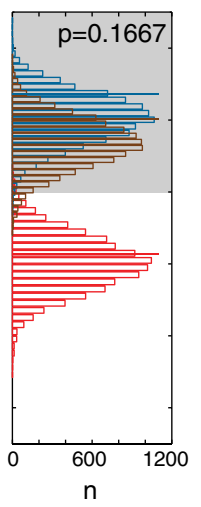

(d)

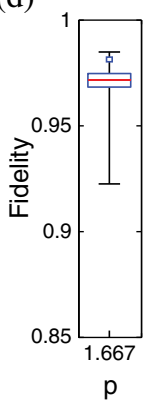

FIG. 4 (color online). (a) Minimum eigenvalue after partial transposition $\lambda^{\mathrm{min}}$ for each bipartition of $\beta_{A B C}$, against a whitenoise admixture $p$. The dash-dotted (dashed) black lines show the theoretical values for infinite counts for the $A \mid B C(C \mid A B$ and $B \mid A C$ ) bipartitions, respectively. Error bars represent 1 standard deviation of the distributions described in (b). (b) $\lambda^{\min }$ for $p=$ 0.1667 , with experimental data (solid lines) and Monte Carlo distribution (histogram) based on a population of 10000 tomographic reconstructions with Poissonian variation of the measured counts. (c) Proportion of the Monte Carlo population for which only bipartition $\beta_{A \mid B C}$ has $\lambda^{\text {min }}<0$. The solid line is a guide to the eye constructed by ideally adding white noise to the $p=0$ experimental state. (d) Box-and-whisker plot representing the fidelity distribution of the theoretical state with the Monte Carlo population for $p=0.1667$; the whiskers indicate maximum and minimum values. The data point represents the fidelity of the experimentally obtained state with the ideal one. See Sec. III of [27] for a discussion of statistical effects of limited photon counts in our experiment. 
entanglement is strictly smaller than the communicated amount of discord, confirming Eq. (2)

A key question to address is the potential advantage of the protocol over other communication-based strategies for entanglement distribution. In this context, it is worth stressing that Alice and Bob will always do better by directly sharing maximally entangled states, if those are available [15]. However, given noisy resources to start with - a reasonable assumption in any practical settingwe can identify regimes under which the distribution of entanglement via separable carriers is a winning strategy. In the Sec. V of [27], we show that for depolarizing and dephasing noise and starting from the paradigm resource embodied by Werner states, the protocol demonstrated here outperforms the direct sharing of entanglement. More specifically, we show that with such resources and under the action of the above quantum channels, the amount of distributed entanglement is higher using the protocol based on communication of separable states. Remarkably, in certain cases, only this scheme is able to distribute entanglement, thus demonstrating its practical value as an effective means to distribute entanglement across a network.

The fundamental insight that an important physical quantity can be increased in an experimental setting without transmitting that quantity reveals yet another counterintuitive aspect of quantum mechanics. We demonstrated that distillable entanglement can indeed be distributed between remote parties who exchange only unentangled carriers. The success of our protocol is confirmed by the unambiguously entangled nature of the $A \mid B C$ bipartition and the separability of the other two. An equally interesting albeit weaker statement on entanglement distribution via bound-entangled states would be possible by having a $C \mid A B$ bipartition that has a positive partial transposition, but is not separable. We have shown the robustness of the protocol to noise and the existence of experimentally relevant conditions under which distributing entanglement using a separable information carrier is indeed more advantageous than communicating entanglement between remote nodes of a network.

We would like to thank K. Kristinsson, P. Raynal, and K. Życzkowski for discussions. M.Z. and T.P. are supported by the National Research Foundation and Ministry of Education in Singapore, by the start-up grant of Nanyang Technological University, and NCN Grant No. 2012/05/E/ ST2/02352. M.P. thanks the U.K. EPSRC for financial support through a Career Acceleration Fellowship and a grant under the "New directions for EPSRC research leaders" initiative; A. F. and M. P. A. acknowledge support from Australian Research Council Discovery Early Career Grants No. DE130100240 and No. DE120101899, respectively; and A.G.W. acknowledges support from a UQ Vice-Chancellor's Senior Research Fellowship. This work was supported in part by the Centres for Engineered Quantum Systems (CE110001013) and for
Quantum Computation and Communication Technology (CE110001027).

Note added.-Recently, we became aware of an independent demonstration of the phenomenon discussed here based on continuous-variable systems [28,29]. These implementations make explicit use of the availability, in the Gaussian continuous-variable scenario, of necessary and sufficient criteria for the inseparability of tripartite mixed states. The lack of similar tools in the discretevariable case addressed in our work required the extra analysis reported in Ref. [27]. Moreover, differently from our protocol, the scheme realized in Ref. [29] required the exchange of classical communication beside the communication of separable information carriers.

*a.fedrizzi@uq.edu.au

tomasz.paterek@ntu.edu.sg

[1] B. Groisman, S. Popescu, and A. Winter, Phys. Rev. A 72, 032317 (2005).

[2] M. Pawłowski, T. Paterek, D. Kaszlikowski, V. Scarani, A. Winter, and M. Żukowski, Nature (London) 461, 1101 (2009).

[3] T. K. Chuan, J. Maillard, K. Modi, T. Paterek, M. Paternostro, and M. Piani, Phys. Rev. Lett. 109, 070501 (2012).

[4] R. Horodecki, P. Horodecki, M. Horodecki, and K. Horodecki, Rev. Mod. Phys. 81, 865 (2009).

[5] C. H. Bennett, G. Brassard, C. Crepeau, R. Jozsa, A. Peres, and W. K. Wootters, Phys. Rev. Lett. 70, 1895 (1993).

[6] A. K. Ekert, Phys. Rev. Lett. 67, 661 (1991).

[7] H. Buhrman, R. Cleve, S. Massar, and R. de Wolf, Rev. Mod. Phys. 82, 665 (2010).

[8] C. H. Bennett and S. J. Wiesner, Phys. Rev. Lett. 69, 2881 (1992).

[9] T. S. Cubitt, F. Verstraete, W. Dür, and J. I. Cirac, Phys. Rev. Lett. 91, 037902 (2003).

[10] A. Streltsov, H. Kampermann, and D. Bruß, Phys. Rev. Lett. 108, 250501 (2012).

[11] L. Henderson and V. Vedral, J. Phys. A 34, 6899 (2001).

[12] H. Ollivier and W. H. Zurek, Phys. Rev. Lett. 88, 017901 (2001).

[13] B. Kraus and J. I. Cirac, Phys. Rev. Lett. 92, 013602 (2004).

[14] M. Paternostro, W. Son, and M. S. Kim, Phys. Rev. Lett. 92, 197901 (2004).

[15] A. Kay, Phys. Rev. Lett. 109, 080503 (2012).

[16] V. Vedral, M. B. Plenio, M. A. Rippin, and P. L. Knight, Phys. Rev. Lett. 78, 2275 (1997).

[17] K. Modi, T. Paterek, W. Son, V. Vedral, and M. Williamson, Phys. Rev. Lett. 104, 080501 (2010).

[18] M. Horodecki, P. Horodecki, R. Horodecki, J. Oppenheim, A. Sen(De), U. Sen, and B. Synak-Radtke, Phys. Rev. A 71, 062307 (2005).

[19] R. Horodecki and M. Horodecki, Phys. Rev. A 54, 1838 (1996).

[20] A. Peres, Phys. Rev. Lett. 77, 1413 (1996). 
[21] G. Vidal and R.F. Werner, Phys. Rev. A 65, 032314 (2002).

[22] M. Horodecki, P. Horodecki, and R. Horodecki, Phys. Rev. Lett. 78, 574 (1997).

[23] N. K. Langford, T. J. Weinhold, R. Prevedel, K. J. Resch, A. Gilchrist, J. L. O'Brien, G. J. Pryde, and A. G. White, Phys. Rev. Lett. 95, 21 (2005).

[24] D. F. V. James, P. G. Kwiat, W. J. Munro, and A. G. White, Phys. Rev. A 64, 052312 (2001).

[25] J. Lavoie, R. Kaltenbaek, M. Piani, and K. Resch, Phys. Rev. Lett. 105, 130501 (2010).

[26] M. Horodecki, P. Horodecki, and R. Horodecki, Phys. Rev. Lett. 80, 5239 (1998).
[27] See Supplemental Material at http://link.aps.org/ supplemental/10.1103/PhysRevLett.111.230504 for a detailed analysis of the techniques used to reveal the separable nature of the experimentally produced states, statistical effects of limited photon counting on entanglement detection, validation of the discord bound, and robustness of the implemented protocol to noise.

[28] C. E. Vollmer, D. Schulze, T. Eberle, V. Handchen, J. Fiurasek, and R. Schnabel, following Letter, Phys. Rev. Lett. 111, 230505 (2013).

[29] C. Peuntinger, V. Chille, L. Mišta, N. Korolkova, M. Förtsch, J. Korger, C. Marquardt, and G. Leuchs, this issue, Phys. Rev. Lett. 111, 230506 (2013). 